\title{
Bioinformatic analysis of PD 1 checkpoint blockade responsive immune microenvironment in severe influenza infection
}

huilin ou

Ningbo lihuili Hospital https://orcid.org/0000-0002-4265-2811

Keda Chen

shulan

Hongcheng Wu

ningbo lihuili hospital

hangping Yao ( $\nabla$ yaohangping@zju.edu.cn )

First Hospital of Zhejiang Province: Zhejiang University School of Medicine First Affiliated Hospital

\section{Research}

Keywords: PD 1/PD L1, influenza, immune checkpoint, immune microenvironment

Posted Date: October 18th, 2021

DOI: https://doi.org/10.21203/rs.3.rs-962677/v1

License: @ (i) This work is licensed under a Creative Commons Attribution 4.0 International License.

Read Full License 
3 Huilin $\mathrm{Ou}^{1}$, Keda $\mathrm{Chen}^{2}$, Hongcheng $\mathrm{Wu}^{1}$, Hangping $\mathrm{Yao}^{3 *}$,

41 Ningbo Medical Centre, Li Huili Hospital affiliated of Ningbo University, Ningbo 315040,

5 China

62 Shulan International Medical College, Zhejiang Shuren University, Hangzhou 310015, China

$7 \quad 3$ State Key Laboratory for Diagnosis and Treatment of Infectious Diseases, First Affiliated

8 Hospital, Zhejiang University School of Medicine, Hangzhou 310003, China

$9 \quad *$ Corresponding author: Hangping Yao

Address: First Affiliated Hospital, Zhejiang University School of Medicine, Hangzhou, China

Tel: 86-574-87236580, Fax: 86-574-87236582, Email: yaohangping@zju.edu.cn

12

Key words: PD-1/PD-L1, influenza, KEGG, Gene

\section{Abstract:}

Background: The programmed cell death 1 (PD-1)/PD-1 ligand 1 (PD-L1) signaling pathway is significantly upregulated in severe influenza virus infection, which impairs the immune system and causes increased tissue inflammation and damage. Blocking this signaling pathway will reduce the damage, lower the virus titer in lung tissue, and alleviate the symptoms of infection to promote recovery. The aim of this study was to identify the key factors and regulatory mechanisms in the PD-1 checkpoint blockade-responsive immune microenvironment in severe influenza infection.

Methods: A BALB/c mouse model of severe influenza A/H1N1 infection was constructed, and whole-transcriptome sequencing of mice treated with PD-1 checkpoint blockade before severe 
A/PR8(H1N1) influenza infection and IgG2a isotype control before infection were performed. Subsequently, the differential expression of nucleic acids between these two groups was analyzed, followed by functional interaction prediction analysis to investigate gene-regulatory circuits.

Results: In total, 84 differentially expressed (dif) mRNAs, 36 dif-microRNAs (miRNAs), 90 dif-IncRNAs (long noncoding RNAs), and 22 dif-circRNAs (circular RNAs) were found in PD-1 antagonist treated A/PR8(H1N1) influenza infection lung compared with the controls (IgG2a isotype control treated before infection). In spleens between the above two groups, 45 dif-mRNAs, 36 dif-miRNAs, 57 dif-lncRNAs, and 24 dif-circRNAs were identified. Direct function enrichment analysis of dif-mRNAs and dif-miRNAs showed that these genes were mainly involved in myocardial damage related to viral infection, mitogen activated protein kinase (MAPK) signaling pathways, RAP1 (Ras-related protein 1) signaling pathway, and Axon guidance. Finally, 595 interaction pairs were obtained for the lungs and 462 interaction pairs for the spleens were obtained in the competing endogenous RNA (ceRNA) complex network, in which the downregulated mmu-miR-7043-3p and Vps39-204 were enriched significantly.

Conclusions: The present study provided a basis for the identification of potential pathways and hub genes that might be involved in the PD-1 checkpoint blockade-responsive immune microenvironment in severe influenza infection.

Key words: PD-1/PD-L1, influenza, immune checkpoint, immune microenvironment

\section{Background}

Programmed cell death 1 (PD-1) is a negative immune checkpoint molecule that downregulates T cell activity after binding with its ligand, PD-1 ligand 1 (PD-L1), during immune 
responses. In chronic infections or tumors, PD-1 overexpression after lasting antigen-exposure will impair the immune response to clear the pathogens or degenerate cells ${ }^{1}$. PD-1 blockade can restore $\mathrm{T}$ cell function, and is already used as a successful therapy in multiple cancer treatments ${ }^{2-3}$. The role of the PD-1/PD-L1 pathway in inhibiting immunity during chronic infections is well established $^{4}$. Recently, its role in acute infections has aroused research attention ${ }^{5}$. The PD-1/PD-L1 pathway has been proven to not only dampen $\mathrm{T}$ cell responses and restrain memory $\mathrm{T}$ cells during some acute infections, but also limits the function of dendritic cells (DCs), macrophages, and $\mathrm{T}$ cell independent $\mathrm{B}$ cell responses. The precise mechanism by which the PD-1/PD-L1 pathway regulates immune responses during acute infection remains unclear.

Influenza virus, especially influenza A virus (IAV) infection, is a huge challenge to global public health, which, because of its high morbidity and mortality, and extremely high antigen mutation rate, has the possibility of causing epidemic outbreaks and even human-to-human transmission $^{6}$. Severe infections often cause fatal pneumonia, which quickly leads to acute respiratory distress syndrome (ARDS) and multiple organ failure. In recent years, studies have proven that acute influenza virus infection, especially severe infections, induce upregulated expression of the PD-1/PD-L1 pathway in an interferon receptor signaling-dependent manner, which leads to degranulation dysfunction and exhaustion of immune cells, especially $\mathrm{CD}^{+} \mathrm{T}$ cells ${ }^{7}$.

The airway epithelium is the first barrier against influenza infection, which participates in host defense by producing cytokines and chemokines, and by regulating expression of surfactant proteins and adapter molecules. Experiments have confirmed that severe influenza virus infection can induce PD-1/PD-L1 signal overexpression and PD- $1^{+}$cell migration to the lung, which plays 
67 an important role in maintaining immune homeostasis ${ }^{8-9}$. The spleen is the largest secondary immune organ and combines the innate and adaptive immune systems, which are important for antibacterial and antifungal immune reactivity. The spleen is a highly organized lymphoid compartment that removes blood-borne microorganisms and cellular debris. PD-1 and PD-L1 expression are high in the $\operatorname{spleen}^{10}$ and upregulation of PD-1 expression correlated well with reduced gamma interferon (IFN- $\gamma$ ) and tumor necrosis factor (TNF) production after virus inoculation.

The transcriptome reflects tissue activity at a given point in time, thus transcriptome expression studies provide an unbiased approach to investigate the PD-1 checkpoint blockade-responsive immune microenvironment during severe influenza infection. RNA sequencing (RNA-Seq) is a next-generation DNA sequencing method that determines the sequences of mRNAs $^{11}$, and has obvious advantages over microarray sequencing. RNA-seq identifies transcription initiation sites and new splicing variants, which makes it possible to precisely determine the exon and splicing isoform expression and to understand the complexity of eukaryotic transcriptomes comprehensively ${ }^{12}$.

\section{Methods}

BALB/c mice (6 to 7 weeks old) were purchased from Joint Ventures SIPPER-BK Experimental Animal Co. (Shanghai, China). All animals were bred and maintained in specific pathogen-free conditions in accordance with the Care and Use of Laboratory Animals of Zhejiang Province and were approved by the local Ethics Committee. Six mice were divided into two groups: 1 . The isotype control followed by A/PR8(H1N1) infection group (severe infection group, $50 \mu \mathrm{L} 10^{6}$ median tissue culture infectious dose (TCID50) infective dose). 2. PD-1 antagonist followed with 
A/PR8(H1N1) infection group. The PD-1 antagonist comprised an antibody against PD-1 (clone RMP1-14; BioXCell, Lebanon, NH, USA), which was administered via tail vein injection in 200 $\mu \mathrm{g}$ doses on days 1,4 , and 7 before infection. An antibody against IgG2a (clone 2A3; BioXCell) was used as the isotype control. Mice were chemically restrained with 2,2,2-tribromoethanol (avertin) before intranasal challenge with $50 \mu \mathrm{L}$ of $10^{6}$ TCID50 virus diluted in phosphate-buffered saline (PBS). Mice were sacrificed 6 days after virus inoculation and their lungs and spleens were collected.

\section{Library preparation and sequencing for small RNAs}

A total of $3 \mu \mathrm{g}$ RNA per sample was used as input material, and sequencing libraries were generated using an NEB Next®Multiplex Small RNA Library Prep Set (NEB, Ipswich, MA, USA). Briefly, the NEB 3' SR Adaptor was ligated to the 3' end of microRNAs (miRNA), small interfering RNAs (siRNAs) and PIWI-interacting RNAs (piRNAs), then the SR RT Primer hybridized to the excess of 3' SR Adaptor and transformed the single-stranded DNA adaptor into a double-stranded DNA molecule. PCR amplification was performed, and then the amplicons were purified. DNA fragments corresponding to $140 \sim 160 \mathrm{bp}$ were recovered and dissolved. Finally, library quality was assessed on an Agilent Bioanalyzer 2100 system (Agilent, Santa Clara, CA, USA) using DNA High Sensitivity Chips.

The clustering of samples was performed on a cBot Cluster Generation System using TruSeq SR Cluster Kit v3-cBot-HS (Illumina, San Diego, CA, USA). After cluster generation, the library preparations were sequenced on an Illumina Hiseq 2500/2000 platform and 50 bp single-end reads were generated. 

the reference, and the modified software mirdeep2 and sRNA-tools-cli were used to obtain the potential miRNA and draw the secondary structures. The software miREvo and mirdeep 2 were integrated to predict novel miRNAs. We followed the following priority rule: Known miRNA > rRNA $>$ tRNA $>$ snRNA $>$ snoRNA $>$ repeat $>$ gene $>$ NAT-siRNA $>$ gene $>$ novel miRNA $>$ ta-siRNA to make every unique small RNA mapped to only one annotation. The known miRNAs used miFam.dat (http://www.mirbase.org/ftp.shtml) to look for families; novel miRNA precursors were submitted to Rfam (http://rfam.sanger.ac.uk/search/) to look for Rfam families. Predicting the target genes of the miRNAs was performed using miRanda. Differential expression analysis was performed using the DESeq R package (1.8.3) with a P-value of 0.05 set as the threshold. The P-values was adjusted using the Benjamini \& Hochberg method. differentially expressed miRNAs. We used KOBAS software to test the statistical enrichment of the target gene candidates in KEGG (Kyoto Encyclopedia of Genes and Genomes) pathways.

\section{Library preparation and sequencing for IncRNAs}

A total of $3 \mu \mathrm{g}$ RNA per sample was used as input material to construct sequencing libraries, which were generated using the rRNA-depleted RNA by NEB Next ${ }^{\circledR}$ Ultra $^{\mathrm{TM}}$ Directional RNA Library Prep Kit for Illumina®. The clustering of samples was performed on a cBot Cluster 
131

132

133

134

135

136

137

\section{Data analysis of IncRNAs}

Clean data were obtained by removing reads containing adapter or poly-N sequences. An index of the reference genome was built using bowtie 2 v2.2.8 and paired-end clean reads were aligned to the reference genome using HISAT2 v2.0.4. The mapped reads of each sample were assembled using StringTie (v1.3.1) in a reference-based approach. We used phyloFit to compute phylogenetic models for conserved and non-conserved regions and then submitted the model and HMM transition parameters to phyloP to compute a set of conservation scores of lncRNAs and coding genes. We clustered the genes from different samples using weighted gene co-expression network analysis (WGCNA) to search for common expression modules and then analyzed their function through functional enrichment analysis. Transcripts with P-adjust $<0.05$ were assigned as differentially expressed. GO enrichment analysis and KEGG pathway enrichment analysis were performed as above.

\section{Results}

\section{Differential Expression Analysis}

In the differential expression analysis of lungs (Figure 1), 85 differentially expressed mRNAs (dif-mRNAs) were obtained, of which 76 were upregulated and 9 were downregulated; 36 differentially expressed miRNAs (dif-miRNAs) were identified, of which 19 were upregulated and 17 were downregulated; 90 differentially expressed lncRNAs (dif-lncRNAs) were obtained, including 70 upregulated and 20 downregulated; and 22 differentially expressed circRNAs (dif-circRNAs) were found, of which 13 were upregulated and 9 were downregulated.

In the spleen data, 45 dif-mRNAs were obtained, of which 18 were upregulated and 27 were 

and 24 dif-circRNAs were found, of which 18 were upregulated and 6 were downregulated.

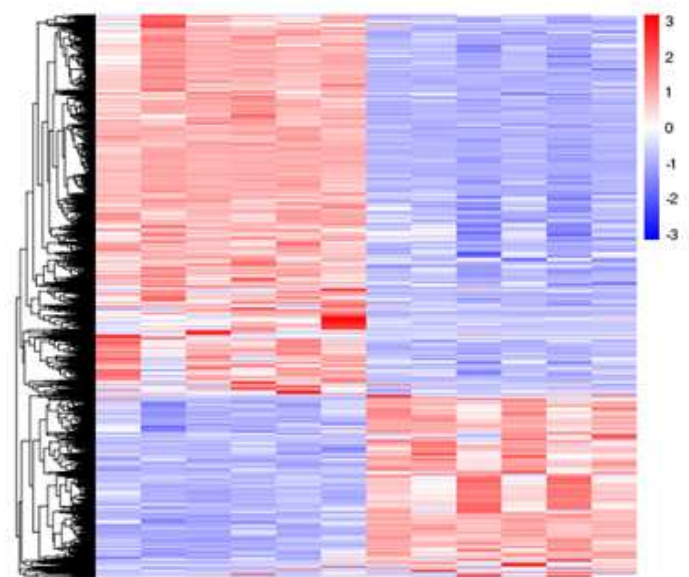

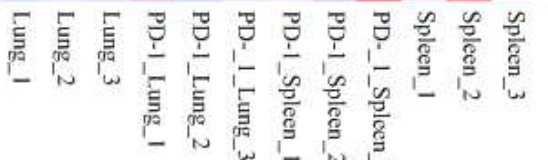

(A)

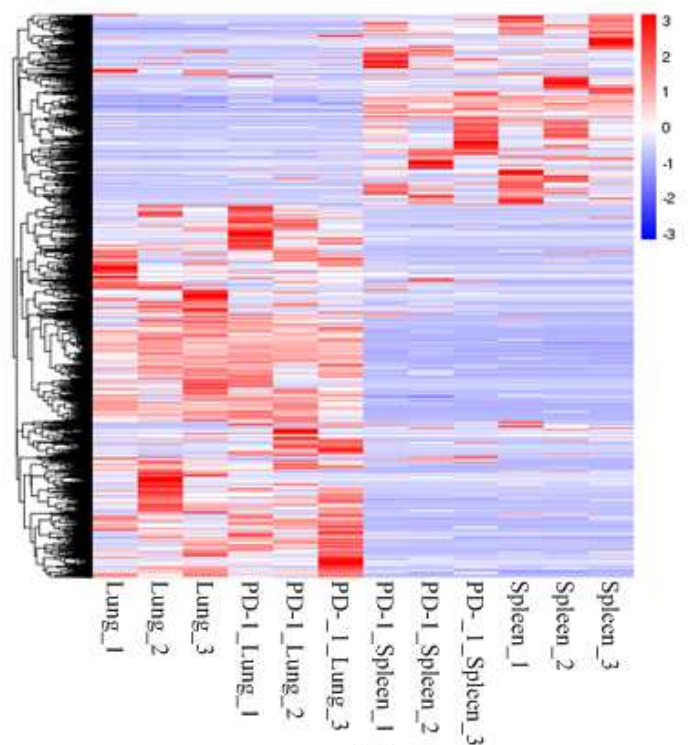

(C)

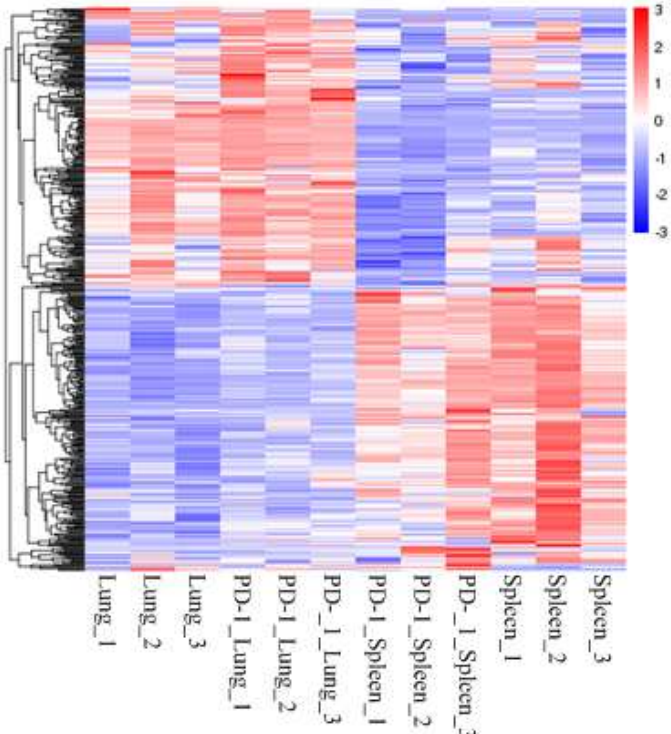

(B)

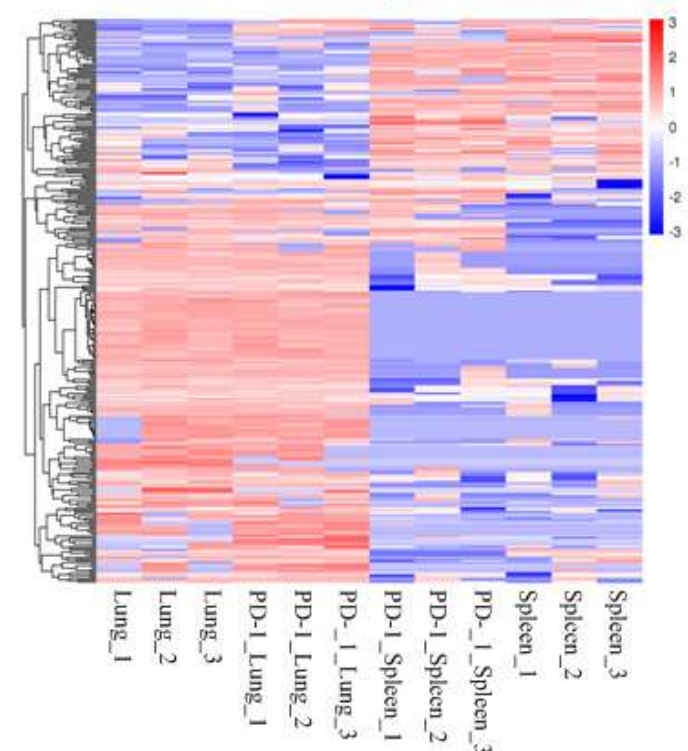

(D)

Figure 1. Heatmaps of differentially expressed nucleic acids. Heatmaps of differentially expressed mRNAs (A), differentially expressed miRNAs (B), differentially expressed lncRNAs (C), and differentially expressed circRNAs (D) of lungs and spleens of the following groups: PD-1 antagonist treatment followed by A/PR8(H1N1) infection group $v s$. isotype control followed by 
A/PR8(H1N1) infection group. Red indicates upregulation, and blue indicates downregulation.

\section{Functional enrichment analysis of dif-mRNAs and dif-miRNAs in lungs and spleens}

KEGG and GO analyses were used to investigate the functional associations of gene expression changes. Targeted genes of dif-mRNA and dif-miRNAs of lungs and spleens of the two groups: PD-1 antagonist followed with A/PR8(H1N1) infection group vs. Isotype control followed with A/PR8(H1N1) infection were predicted (Figure 2 and Figure 3 ). The gene lists used in the dif-mRNAs analysis contained 18455 and 17818 genes for lungs and spleens, respectively. 1290 and 1290 genes were analyzed for lungs and spleens for dif-miRNAs. For GO, biological process, cellular component, and molecular function were selected as the annotation categories for clustering. Once the tool identified enriched ontologies for a particular gene list, it clusters those that have a statistically significant overlap in terms of their constituent genes. The dif-mRNAs were enriched in 81 pathways in lungs and 36 pathways in spleens. dif-miRNAs were enriched in 274 pathways in lungs and 273 pathways in spleens. There was little degree of overlap of dif-mRNAs and dif-miRNAs in lungs between the most enriched clusters. The most enriched clusters of dif-mRNAs of lungs were related to muscle and heart biological behavior. More than $85 \%$ of the dif-miRNAs enriched clusters in lungs and spleens overlapped with each other, including localization, metabolic process, positive regulation of metabolic process, and regulation of molecular function in the biological process category; intracellular part, cytoplasm, intracellular, and membrane-bounded organelle in the cellular component category; and protein binding, enzyme binding, and molecular function regulator in the molecular function category.

Functional Enrichment analysis of mRNAs and miRNAs in lungs and spleens obtained from severe IAV infection mice treated with anti-PD-1 antibody clearly highlighted myocardial damage 
(Ras-related protein 1) signaling pathway, and Axon guidance.

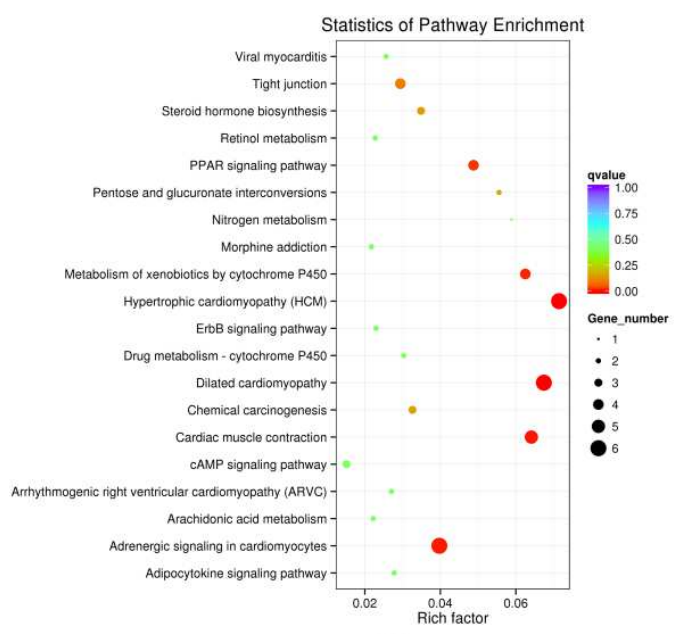

(A)

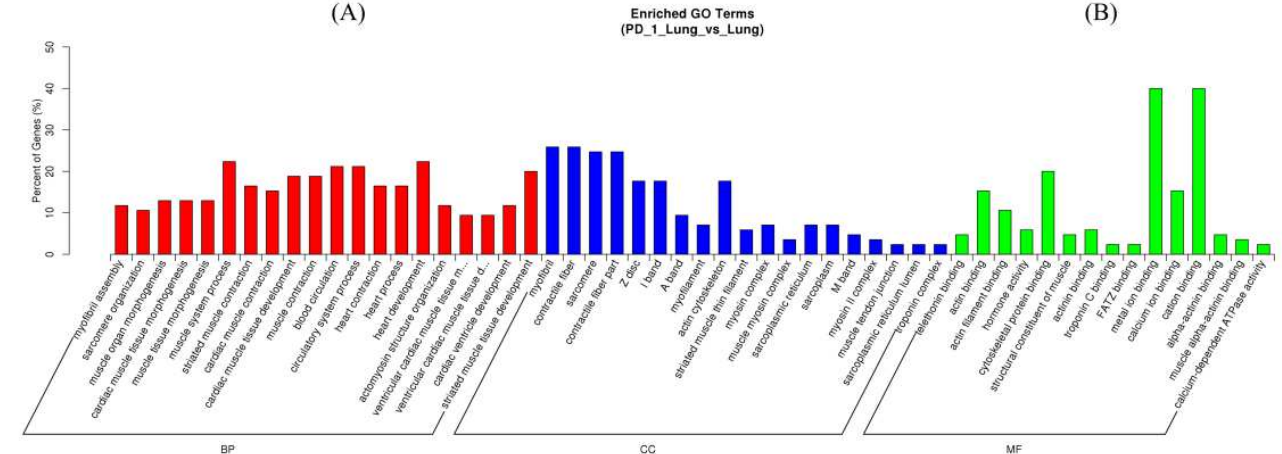

(C)

Enriched Go Terms
(PD_1_LungusLung)

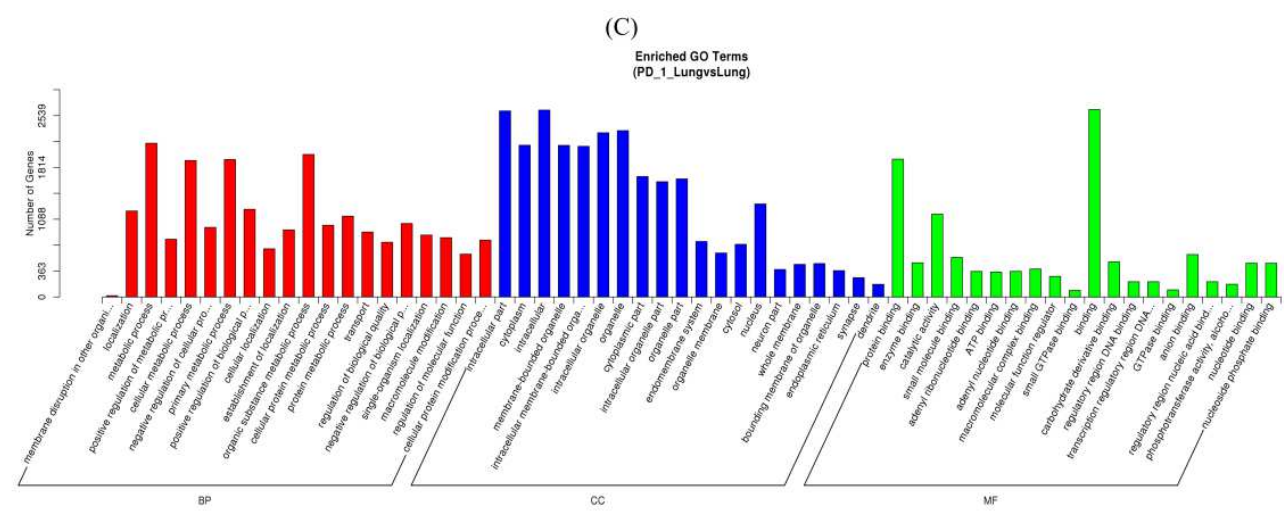

(D)

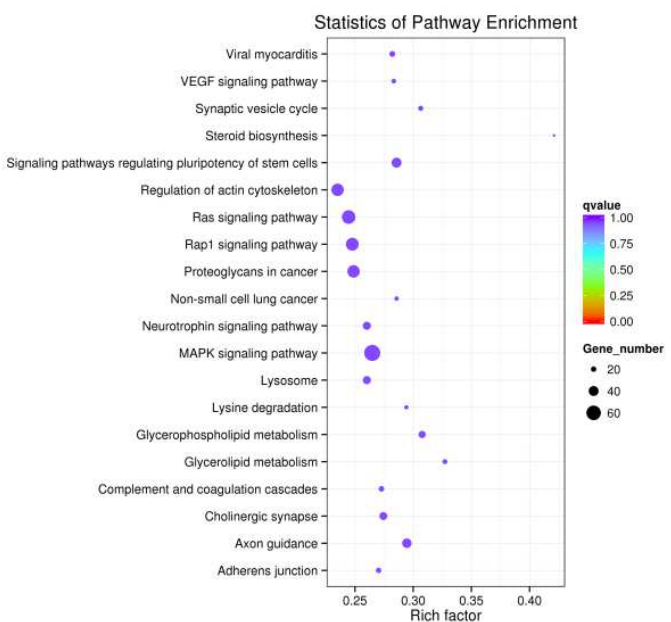

(B)

Figure 2. Gene Ontology (GO) and KEGG pathway analysis of dif-mRNAs and dif-miRNAs in the lungs. The top 20 pathways and GO terms (BP (Biological Process), CC (cellular component), and MF (molecular function)) enriched by dif-mRNAs and dif-miRNAs of lungs of the following groups: PD-1 antagonist treatment followed by A/PR8(H1N1) infection group vs. isotype control followed by A/PR8(H1N1) infection group. (A) Top 20 pathways enriched by dif-mRNAs. (B) 

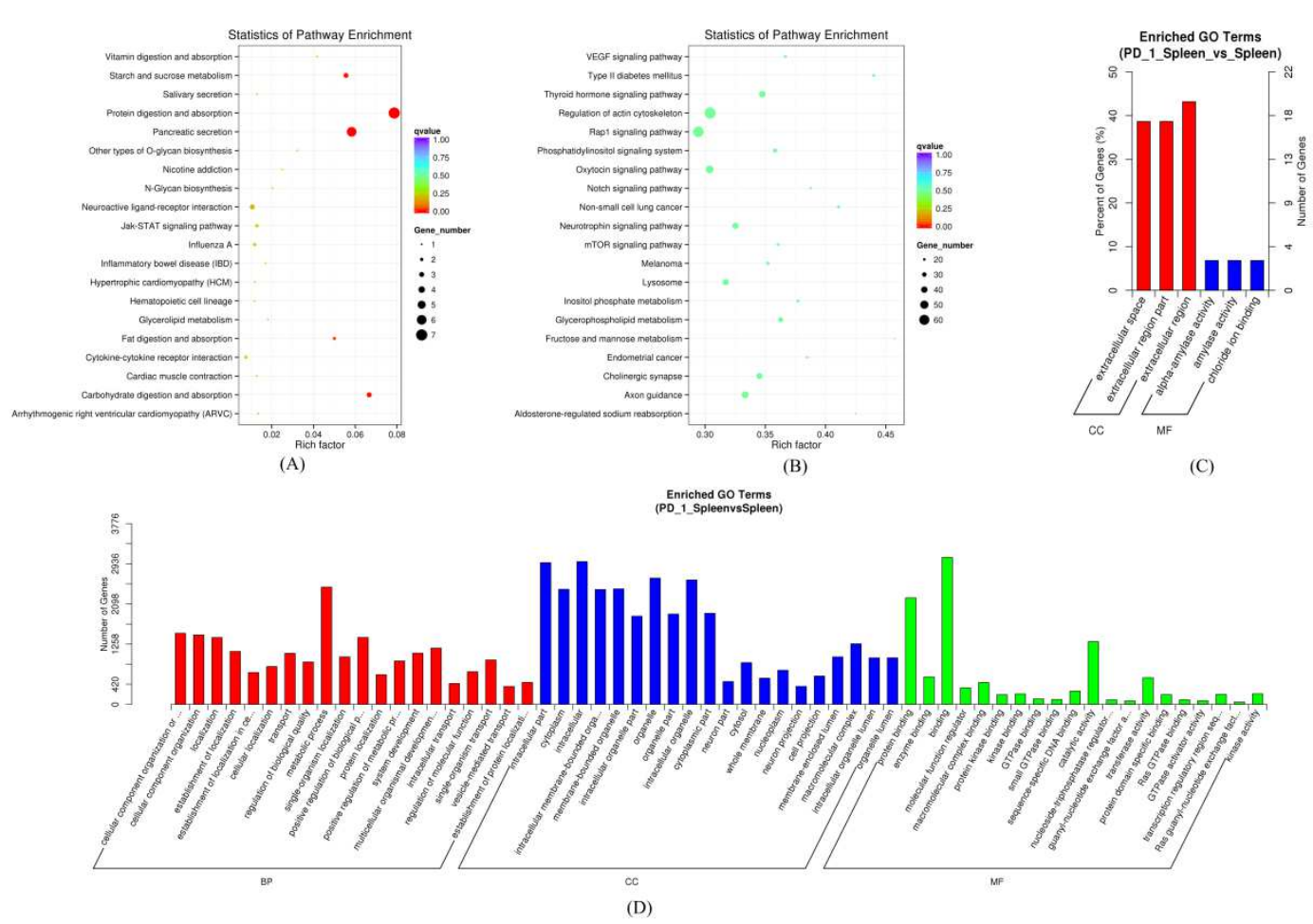

Figure 3. GO and KEGG pathway analysis of dif-mRNAs and dif-miRNAs in spleens. 
198

199

200

201

202

203

204

205

206

207

pathways enriched by dif-miRNAs (C) Top 20 GO terms enriched by dif-mRNAs (D) Top 20 GO terms enriched by dif-miRNAs.

\section{Protein-Protein Interaction (PPI) Network}

The PPI network based on dif-mRNAs between lungs consisted of 24 nodes and 24 interaction pairs (Figure 4). Top 10 KEGG pathways enriched by genes in the PPI network were significantly involved in heart damage. The PPI network based on dif-mRNAs between spleens consisted of 10 nodes and 10 interaction pairs (Figure 5).

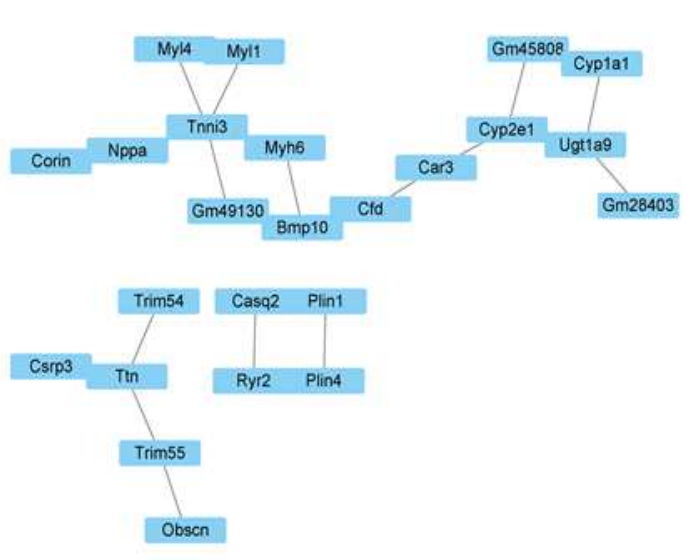

(A)

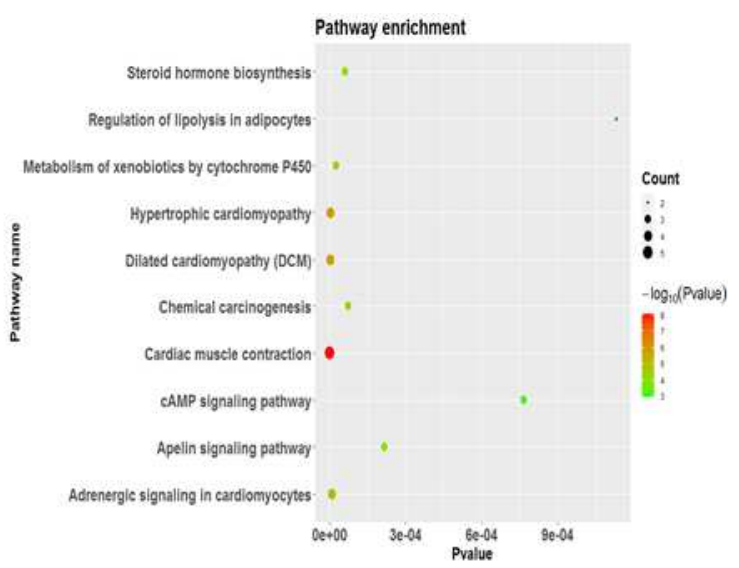

(B)

Figure 4. Protein-Protein Interaction (PPI) analysis in the lungs. (A) PPI network of dif-mRNAs in the lungs. (B) Top 10 KEGG pathways enriched by genes in the lung PPI network.

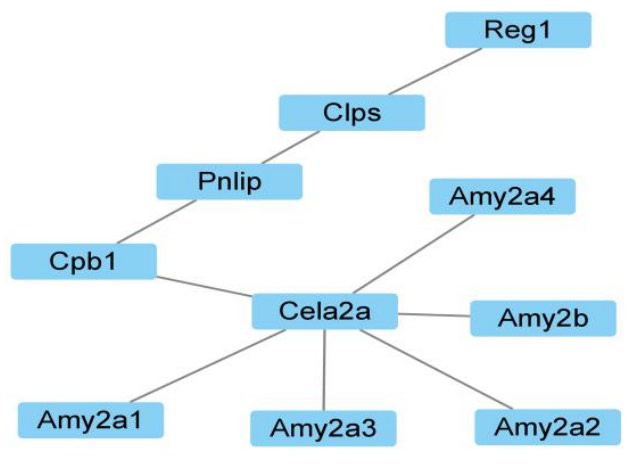

(A)

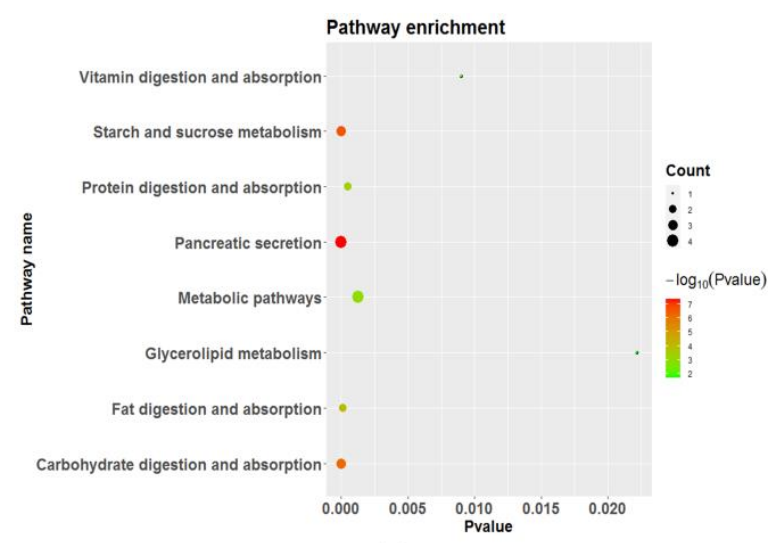

(B)

Figure 5. Protein-Protein Interaction (PPI) analysis in the spleens. (A) PPI network of dif- 

mRNAs in spleens. (B) Top 8 KEGG pathways enriched by genes in the spleens PPI networks. target genes (Figure 6 and Figure 7). The dif- IncRNA target genes were enriched in 100 pathways

214 in lungs and 154 pathways in spleens. The dif- circRNA target genes were enriched in 20

215 pathways in lungs and 14 pathways in spleens. There was a little degree of overlap of lncRNAs

216 and circRNAs in lungs and spleens between the most enriched clusters except for Hypertrophic

217 cardiomyopathy, MAPK signaling pathway, and the AMP-activated protein kinase (AMPK)

218 signaling pathway. 


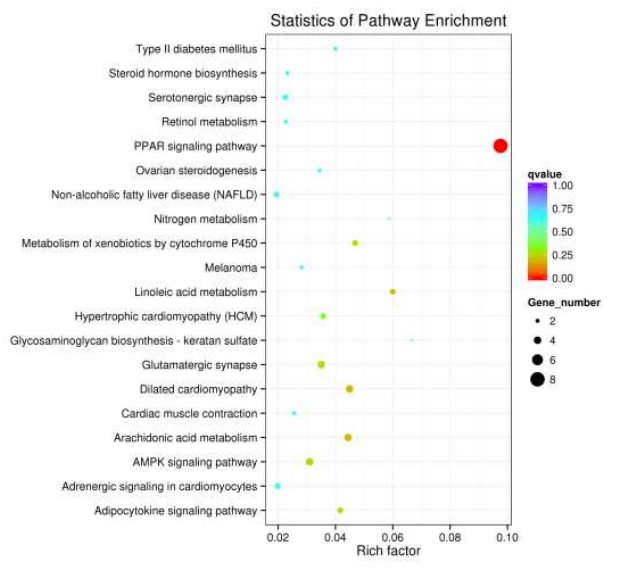

(A)

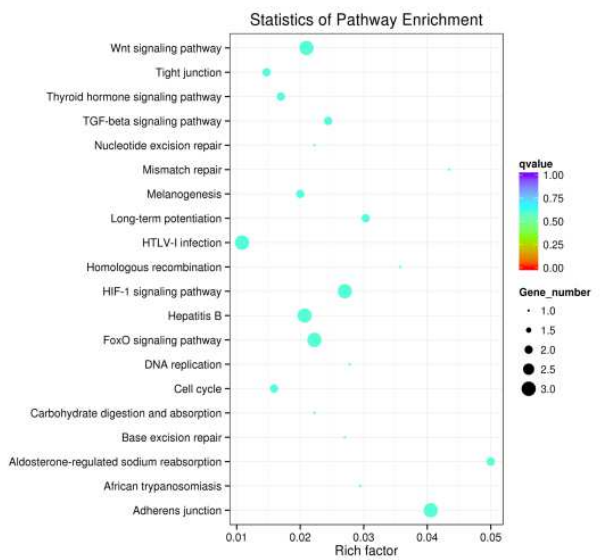

(B)

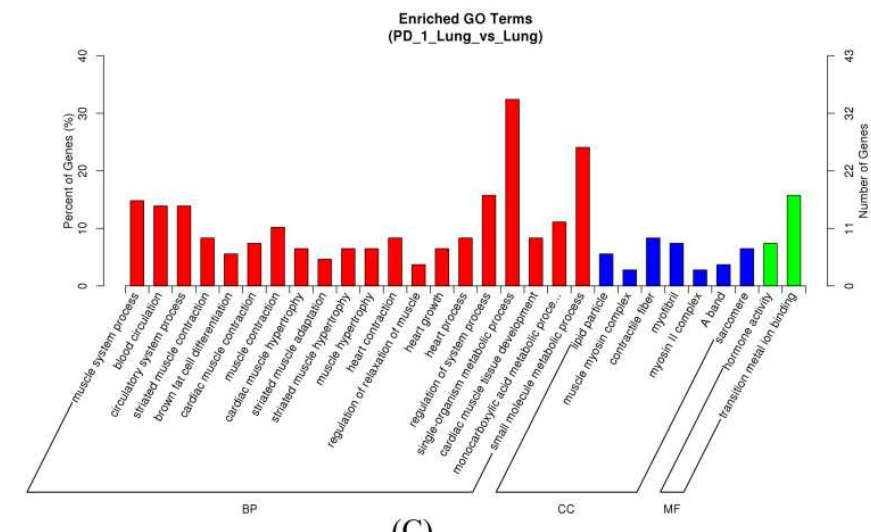

(C)

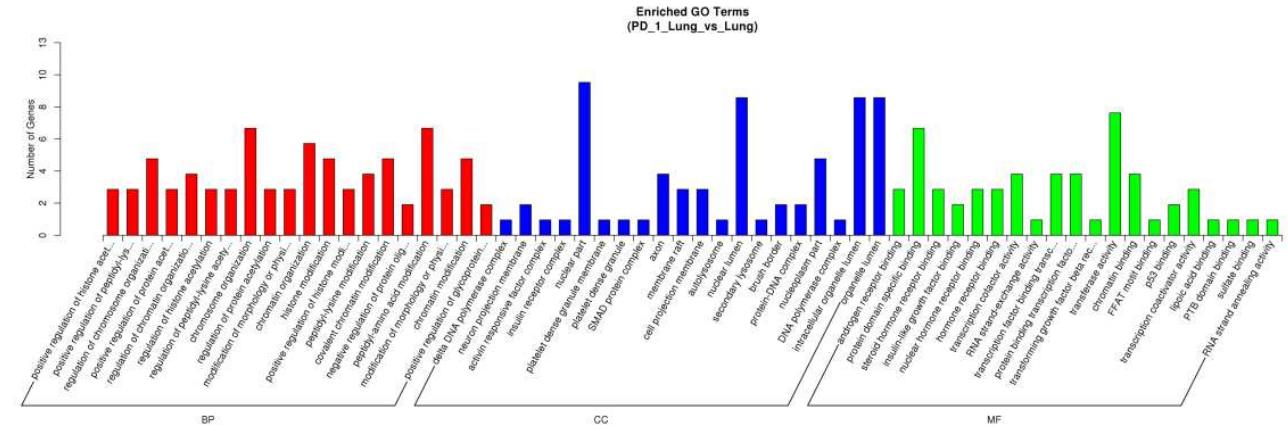

(D)

Figure 6. Analysis of GO and KEGG pathways of dif- lncRNAs and dif-circRNAs of the lungs.

221 Top 20 pathways and GO terms (BP (Biological Process), CC (cellular component), and MF

222 (molecular function)) enriched by dif- IncRNAs and dif-circRNAs of the lungs (A) Top 20

223 pathways enriched by dif-lncRNAs. (B) Top 20 pathways enriched by dif-circRNAs (C) Top 20

224 GO terms enriched by dif-lncRNAs (D) Top 20 GO terms enriched by dif-circRNAs. 


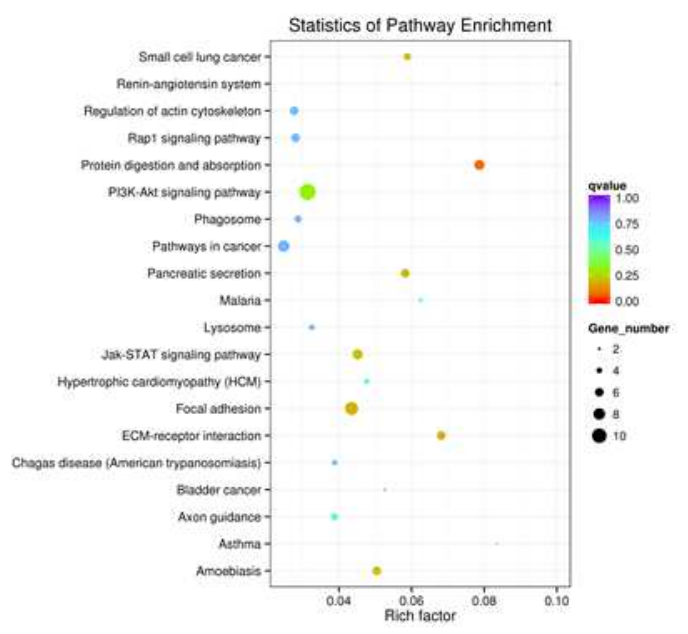

(A)

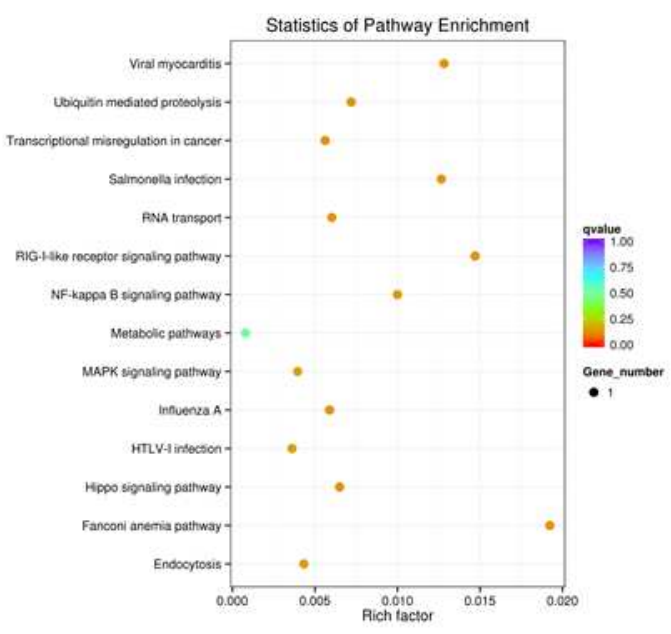

(B)

Enriched Go Terms
(PD_1_Spleen_vs_Spleen)

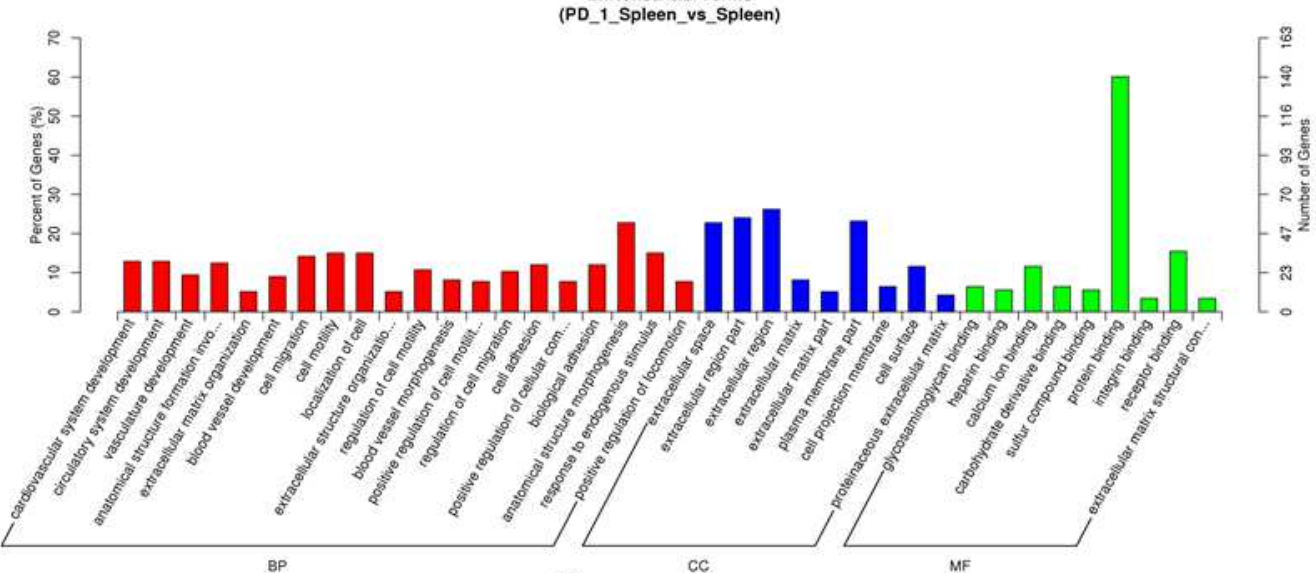

(C)

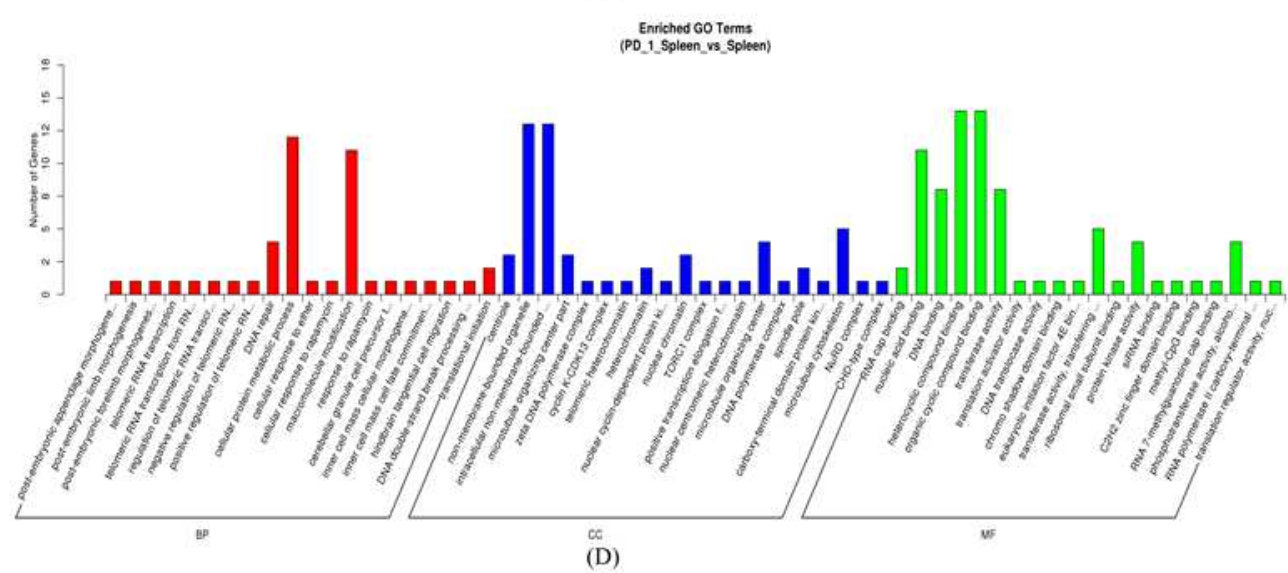

Figure 7. Analysis of GO and KEGG Pathway of dif- lncRNAs and dif-circRNAs of spleens. 

expressed lncRNAs and mRNAs regulated by the same miRNA were screened. In total, 77 upregulated lncRNAs and 9 downregulated lncRNAs, 5 upregulated and 5 downregulated mRNAs, and 2 upregulated and 5 downregulated miRNAs. In spleens, 131 lncRNA-miRNA-mRNA interactions were finally obtained (Figure 9), including 29 upregulated lncRNAs and 26 downregulated lncRNAs, 17 upregulated and 8 downregulated mRNAs, and 5 upregulated and 4 downregulated miRNAs. comprising 2 upregulated circRNAs, 2 upregulated mRNAs, and one downregulated miRNA. In including 6 upregulated circRNAs and 1 downregulated circRNA, 16 upregulated mRNAs and 2 


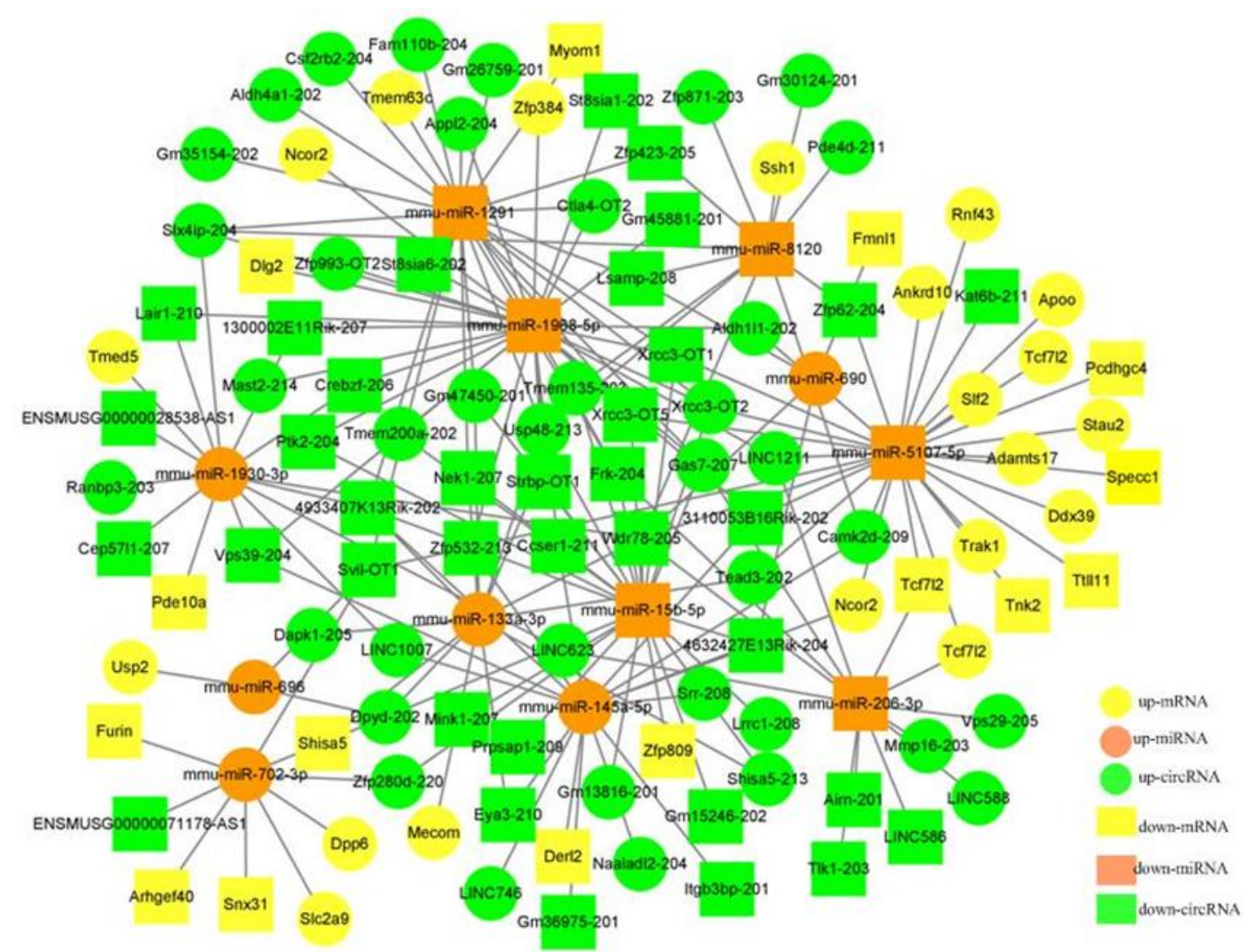

247 Figure 8. The lncRNA-miRNA-mRNA network of the lungs. Circles represent upregulation

248 and rectangles represent downregulation. mRNAs, miRNAs, and lncRNAs in the network are

249 presented in yellow, orange, and green, respectively. 


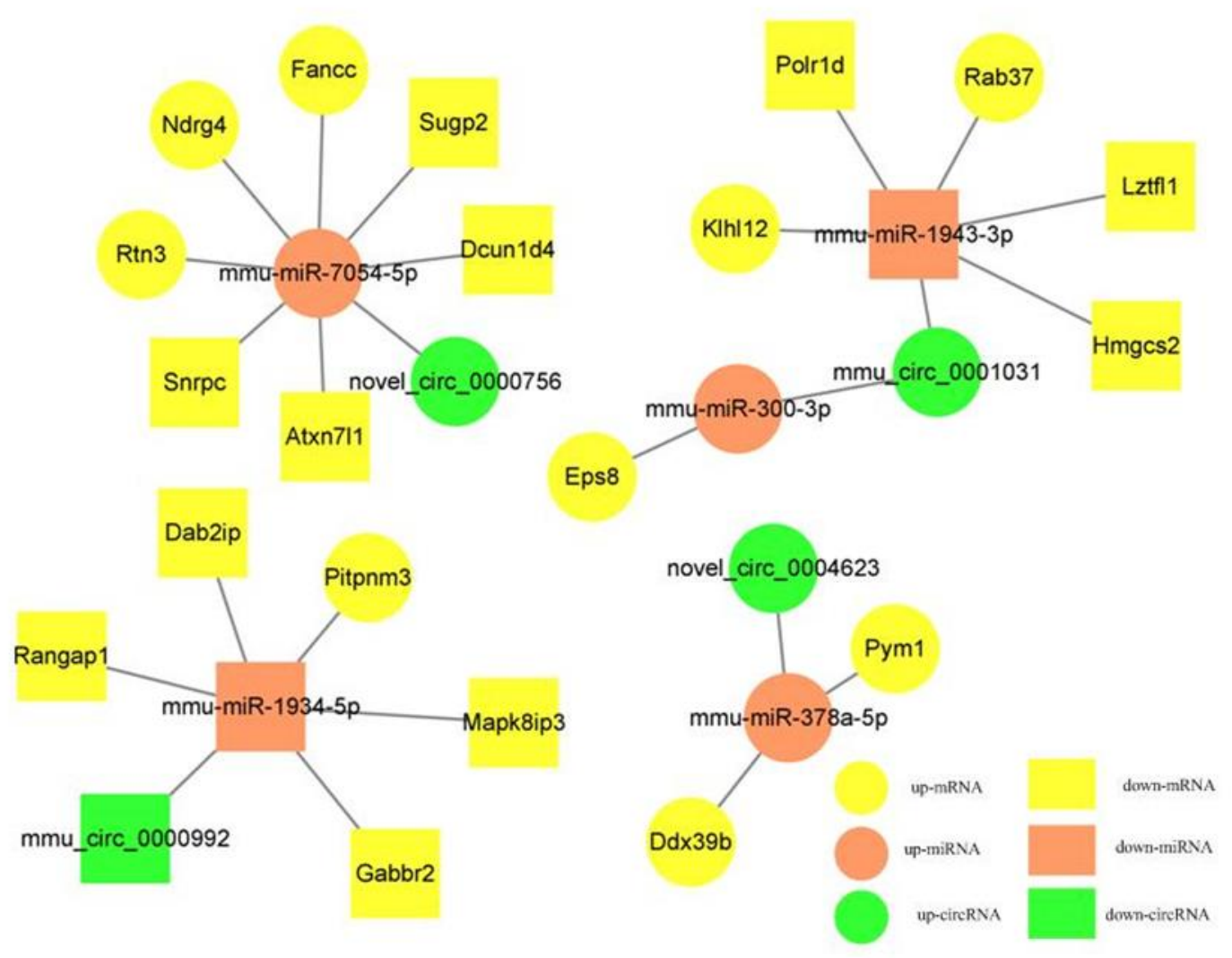

251 Figure 9. The lncRNA-miRNA-mRNA Network of the spleens. Circles represent upregulation

252 and rectangles represent downregulation. mRNAs, miRNAs, and lncRNAs in the network are

253 presented in yellow, orange, and green, respectively. 


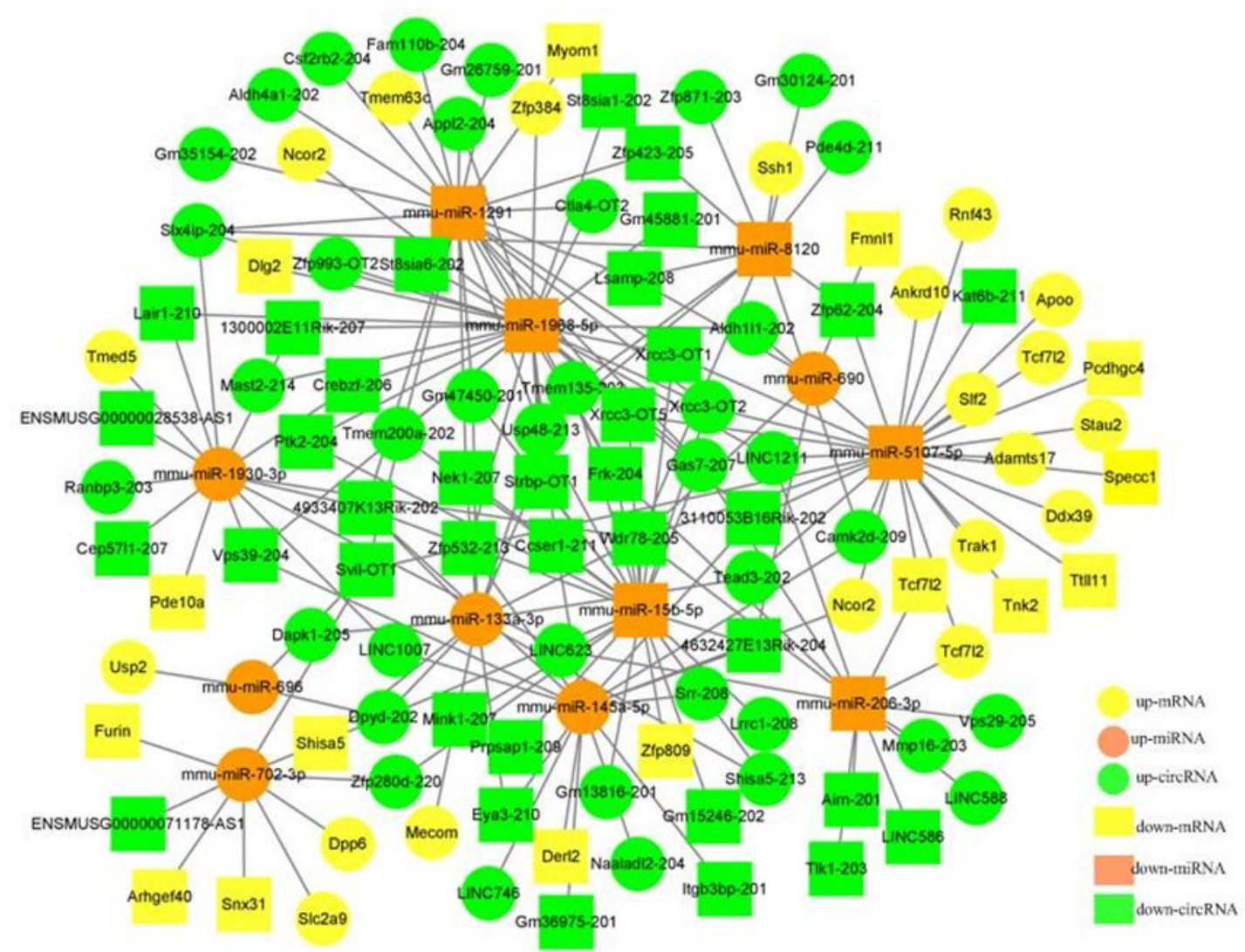

255 Figure 10. The circRNA-miRNA-mRNA network of the lungs. Circles represent upregulation

256 and rectangles represent downregulation. mRNAs, miRNAs, and circRNAs in the network are

257 presented in yellow, orange, and green, respectively. 


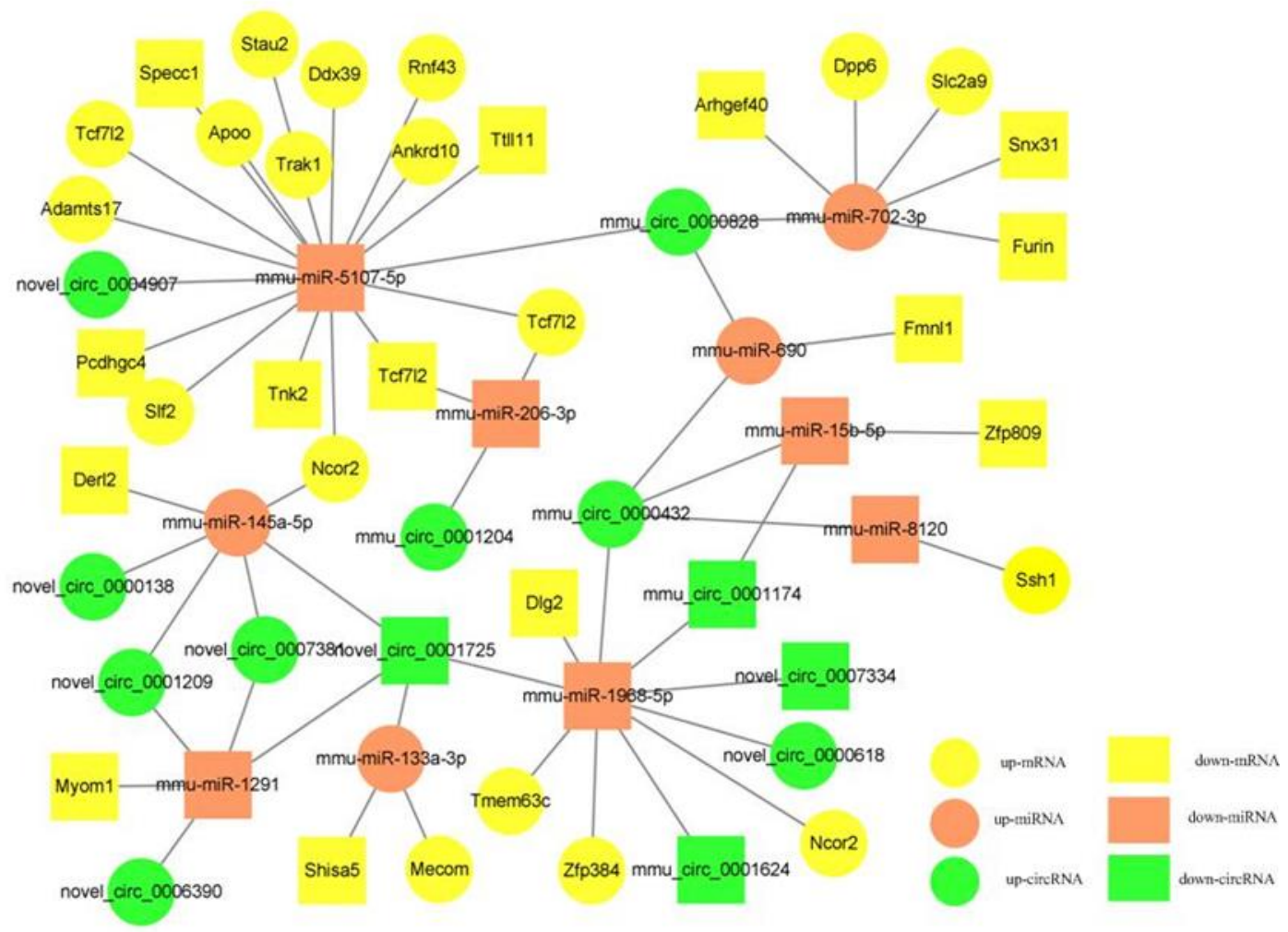

Figure 11. The circRNA-miRNA-mRNA network of the spleens. Circles represent upregulation and rectangles represent downregulation. mRNAs, miRNAs, and circRNAs in the network are presented in yellow, orange, and green, respectively

Further, differentially expressed circRNAs, IncRNAs, and mRNAs that were regulated by the same miRNA were further screened based on the lncRNA-miRNA-mRNA and circRNA-miRNA-mRNA analysis. Finally, 595 interaction pairs were obtained in lungs (Figure 12), comprising 135 upregulated and 63 downregulated mRNAs, 5 upregulated and 5 downregulated miRNAs, 5 upregulated and 2 downregulated circRNAs, and 46 upregulated and

26738 downregulated lncRNAs. There were 462 interaction pairs in spleens (Figure 13), comprising 

network

Figure 12. The Competing Endogenous RNA (ceRNA) network of the lungs. Circles represent upregulation and rectangles represent downregulation. mRNAs, miRNAs, lncRNAs, and circRNAs in the network are presented in yellow, orange, blue, and green, respectively. 


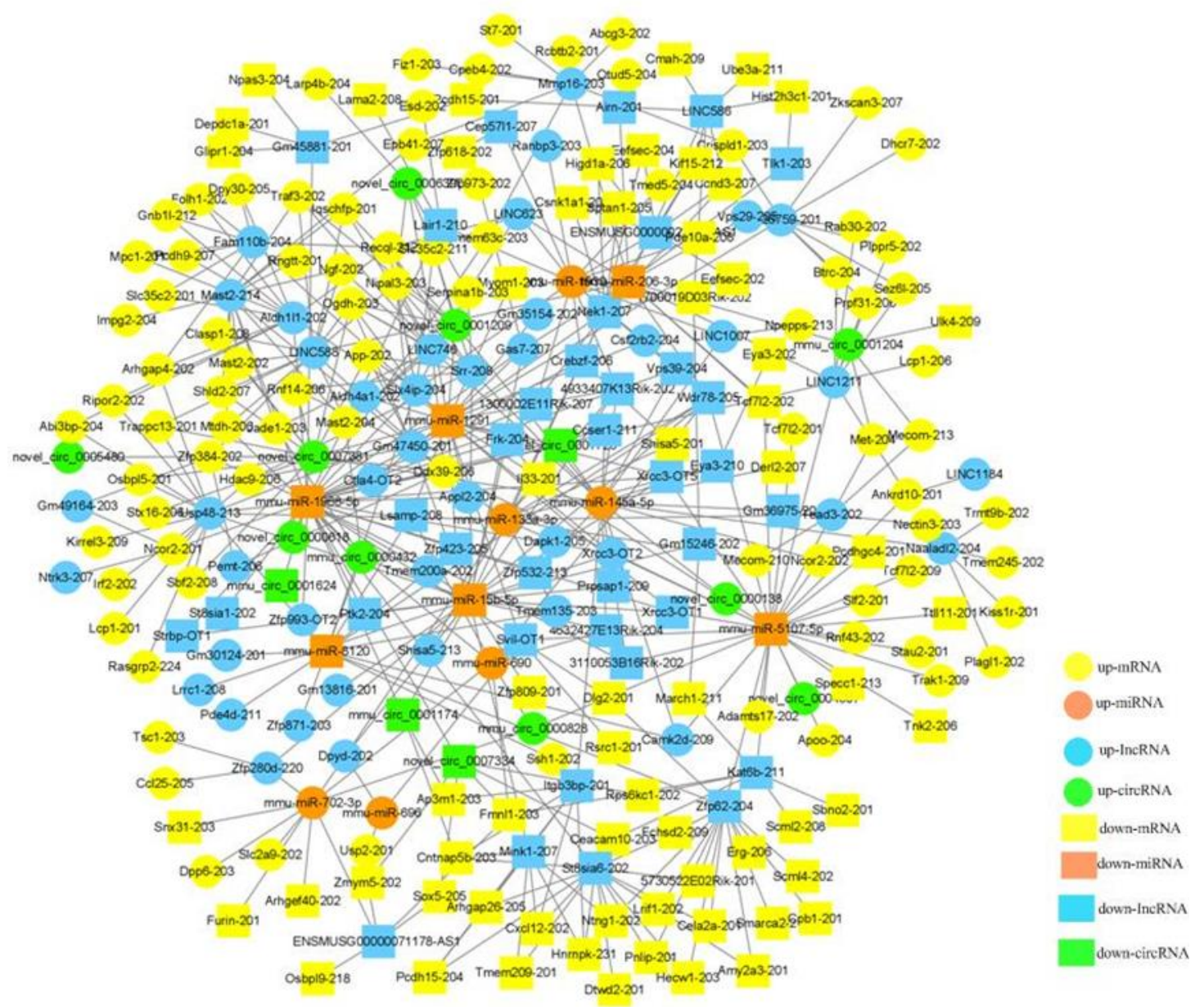

Figure 13. The Competing Endogenous RNA (ceRNA) network of the spleens. Circles

278 represent upregulation and rectangles represent downregulation. mRNAs, miRNAs, lncRNAs, and

279 circRNAs in the network are presented in yellow, orange, blue, and green, respectively.

\section{Discussion}

The PD-1/PD-L1 signaling pathway has important regulatory roles in antiviral immune responses, and PD-1/PD-L1 upregulation is induced by persistent viruses, including human

283 immunodeficiency virus (HIV) ${ }^{13-14}$, hepatitis $\mathrm{C}$ virus $(\mathrm{HCV})^{15}$, and hepatitis B virus $(\mathrm{HBV})^{16}$,

284 which impairs $\mathrm{T}$ cell responses and is unfavorable for virus clearance. Recently, the role of the

285 PD-1/PD-L1 axis during acute virus infection has been further investigated. Upregulated

PD-1/PD-L1 expression induced by severe influenza A virus infection is an important component 
tissue damage, lower virus titers in the lung, and alleviate symptoms of infection to promote recovery $^{7-8,17}$. However, the molecular mechanism of the PD-1 checkpoint in the antivirus immune microenvironment are still not well understood and are thus worthy of in-depth investigation.

Next-generation sequencing, which permits massive sequencing with a much higher throughput, has numerous advantages over traditional sequencing technology, and has been applied in various fields of infection disease research, such as identification of infectious pathogens and exploration of the infection mechanism ${ }^{18-19}$. In this study, by applying whole-transcriptome sequencing, we identified 84 dif-mRNAs , 36 dif-miRNAs, 90 dif-lncRNAs, and 22 dif-circRNAs in PD-1 antagonist treated A/PR8(H1N1) influenza infected lungs compared with those in the controls (IgG2a isotype control treated before infection). In the comparison between the spleen samples from the above two groups, 45 dif-mRNAs , 36 dif-miRNAs, 57 dif-lncRNAs, and 24 dif-circRNAs were identified. Direct functional enrichment analysis on the dif-mRNAs and dif-miRNAs showed that these genes were mainly involved in myocardial damage related to viral infection, MAPK signaling pathways, the RAP1 signaling pathway, and Axon guidance.

Functional Enrichment analysis of mRNA and miRNA in lung and spleen clearly highlighted myocardial damage related to viral infection, and PPI analysis was also significantly enriched for viral heart damage. Influenza virus is an etiological agent of myocarditis, and the relationship between acute respiratory virus infection, especially influenza, and associated viral myocardial damage is greatly underestimated. Many studies have reported that influenza virus infection, especially severe infection, causes fatal myocarditis in humans and experimental animals. Acute 
cardiovascular events even death triggered by influenza was first noted as early as the 1930s.

Several studies have confirmed that acute respiratory infections or influenza-like illnesses were closely related to subsequent acute cardiovascular events ${ }^{20-21}$, and autopsies showed that the majority of the heart was affected in fatal cases during epidemics of influenza ${ }^{22-23}$. Viruses might replicate in the heart of at least $10 \%$ of patients with infection, and pathological injuries include focal infiltration with inflammatory cells in the interstitial and pericardium areas, myocardial edema, and cardiac necrosis. Frequently, both the left and right sides of heart are dilated ${ }^{24}$. The basic treatment is hemodynamic and ventilatory support; however, the use of immunosuppressive or antiviral therapy for fulminant myocarditis of viral etiology is controversial ${ }^{25}$. Our sequencing result suggested that PD-1 antagonist interferes with virus-induced cardiomyocyte damage and might alleviate tissue damage; however, this conclusion needs to be further confirmed in a larger scale animal experiment.

The MAPK signaling pathway plays an important role in regulating cell proliferation, differentiation, invasion, metastasis, and death through phosphorylation activation. The relationship between MAPK signaling pathways and anti-PD-1 antibody in infectious disease has been discussed elsewhere, especially in chronic infection. MAPK activation is an important initiating event in the upregulation of PD-1 in HIV-1-infected cells, and inhibition of this signaling pathway can reduce infection ${ }^{26-27}$. The HA protein of influenza A virus is conserved among strains and subsets, and axon guidance molecules were proven to have a large pentapeptide overlap, thus immune cross-reactivity between influenza HA and axon guidance molecules is possible ${ }^{28-30}$. PD-1 signaling inhibits Rap guanine nucleotide exchange factor 1 (RAPGEF1 also known as C3G) phosphorylation by utilizing SHP-1/2 (also known as protein tyrosine phosphatase non-receptor 
type 6 and type 11), and reduced levels of phosphorylated C3G result in reduced RAP1 activation and adhesion to intercellular adhesion molecule 1 (ICAM-1) to inhibit T-cell adhesion. Several studies suggested that sepsis-induced upregulation of PD-1 has an impact on the motility and migratory capacity of $\mathrm{T}$ lymphocytes by regulating classical inhibitory motif recruitment, activation of the phosphatases SHP-1/2, and signaling through RAP1 ${ }^{31}$.

Additionally, we identified the significant role of downregulated mmu-miR-7043-3p and Vps39-204 in the ceRNA network. Increased expression of mmu-miR-7043-3p was proven to be one of remarkable miRNA signatures of myocardial reductive stress, which is associated with cardiac hypertrophy ${ }^{32}$. Future mechanistic studies are needed to determine the role of miR-7043-3p in PD-1/PD-L1 pathway-associated viral damage in severe influenza infection. VPS39 is a member of the vacuolar tethering complex that promotes late endosome formation, and evidence has shown that silencing VPS39 can increase the proliferation of aged human T cells and memory responses of lysosome-defective $\mathrm{T}$ cells in a mouse viral infection model ${ }^{33}$, and thus might play important roles in antiviral immunity.

\section{Conclusions}

In conclusion, this study explored the molecular mechanism of the PD-1 checkpoint blockade-responsive immune microenvironment during severe influenza infection. Upregulated PD-1/PD-L1 expression-induced by severe IAV infection is an important component of the immunosuppressive microenvironment, and blocking this signaling pathway will regulate the following signal pathways: Myocardial damage related to viral infection, MAPK signaling pathways, Rap1 signaling pathway, and Axon guidance. Downregulated mmu-miR-7043-3p and Vps39-204 were most significantly enriched by PD-1 blockade. However, this study was limited 
354

355

356

357

358

359

360

361

362

363

364

365

366

367

368

369

370

371

372

373

374

375

by a small sample size and limited time points to provide a comprehensive overview of the PD-1 checkpoint blockade-responsive immune microenvironment. Further in vivo validation using a larger scale animal experiment and dynamic functional characterization are needed to delineate the exact mechanistic details.

\section{List of abbreviations}

The programmed cell death 1: PD-1

PD-1 ligand 1: PD-L1

microRNAs: miRNAs

long noncoding RNAs: lncRNAs

circular RNAs: circRNAs

mitogen activated protein kinase : MAPK)

Ras-related protein 1: RAP1

competing endogenous RNA : ceRNA

dendritic cells : DCs

especially influenza A virus: IAV

acute respiratory distress syndrome : ARDS

gamma interferon: IFN $\gamma$

tumor necrosis factor: TNF

RNA sequencing: RNA-Seq

median tissue culture infectious dose: TCID50

phosphate-buffered saline: PBS

small interfering RNAs: siRNAs 
378 Kyoto Encyclopedia of Genes and Genomes: KEGG

379 weighted gene co-expression network analysis: WGCNA

380 Biological Process: BP

381 cellular component: CC

382 molecular function: MF

383 Protein-Protein Interaction : PPI

384 AMP-activated protein kinase : AMPK

385 human immunodeficiency virus: HIV

386 hepatitis $\mathrm{C}$ virus: $\mathrm{HCV}$

387 hepatitis B virus: HBV

388 Rap guanine nucleotide exchange factor 1: RAPGEF1

389 protein tyrosine phosphatase non-receptor type 6 and type 11: SHP-1/2

390 intercellular adhesion molecule 1: ICAM-1

\section{Consent to publication}

$392 \quad$ Not applicable

\section{Conflicts of Interest}

394 The authors declare that they have no competing interests

\section{$395 \quad$ Funding}

396 This work was supported by the Zhejiang Provincial Natural Science Foundation [grant number 
All animal studies were performed in accordance with the Guide for the Care and Use of

Laboratory Animals of Zhejiang Province and were approved by the local Ethics Committee.

401

402

403

404

405

406

407

408

409

410

411

412

\section{Availability of data and material:}

The datasets used or analyzed during the current study are available from the corresponding author on reasonable request.

\section{Authors' Contributions}

HO performed the experiments, analyzed the data, and wrote the first draft. KC and HW reviewed the data and revised the paper. HY designed the experiment and reviewed the data. All Authors read and approved the final version of the article.

\section{Acknowledgements}

The Authors would like to thank Elixigen (http://med2708.yixie8.com/) for their assistance with English language editing.

\section{References}

1. Ayala, A.; Elphick, G. F.; Kim, Y. S.; Huang, X.; Carreira-Rosario, A.; Santos, S. C.; Shubin, N. J.; Chen, Y.; Reichner, J.; Chung, C.-S., Sepsis-induced potentiation of peritoneal macrophage migration is mitigated by programmed cell death receptor-1 gene deficiency. Journal of innate immunity 2014, 6 (3), 325-338.

2. Han, Y.; Liu, D.; Li, L., PD-1/PD-L1 pathway: current researches in cancer. American journal of cancer research 2020, 10 (3), 727.

3. Topalian, S. L.; Hodi, F. S.; Brahmer, J. R.; Gettinger, S. N.; Smith, D. C.; McDermott, D. F.; Powderly, J. D.; Carvajal, R. D.; Sosman, J. A.; Atkins, M. B., 
421

422

423

424

425

426

427

428

429

430

431

432

433

434

435

436

437

438

439

440

441

442

Safety, activity, and immune correlates of anti"CPD-1 antibody in cancer. New England Journal of Medicine 2012, 366 (26), 2443-2454.

4. Sch?nrich, G. n.; Raftery, M. J., The PD-1/PD-L1 axis and virus infections: a delicate balance. Frontiers in cellular and infection microbiology 2019, 9, 207.

5. David, P.; Megger, D. A.; Kaiser, T.; Werner, T.; Liu, J.; Chen, L.; Sitek, B.; Dittmer, U.; Zelinskyy, G., The PD-1/PD-L1 pathway affects the expansion and function of cytotoxic $\mathrm{CD} 8^{+} \mathrm{T}$ cells during an acute retroviral infection. Frontiers in immunology 2019, 10, 54 .

6. Palese, P., Influenza: old and new threats. Nature medicine 2004, 10 (12), S82-S87.

7. Pauken, K. E.; Godec, J.; Odorizzi, P. M.; Brown, K. E.; Yates, K. B.; Ngiow, S. F.; Burke, K. P.; Maleri, S.; Grande, S. M.; Francisco, L. M., The PD-1 pathway regulates development and function of memory $\mathrm{CD}^{+} \mathrm{T}$ cells following respiratory viral infection. Cell reports 2020, 31 (13), 107827.

8. Li, Y. H.; Hu, C. Y.; Cheng, L. F.; Wu, X. X.; Weng, T. H.; Wu, N. P.; Yao, H. P.; Li, L. J., Highly pathogenic H7N9 avian influenza virus infection associated with up-regulation of PD-1/PD-Ls pathway-related molecules. International immunopharmacology 2020, 85, 106558.

9. McNally, B.; Ye, F.; Willette, M.; Fla?o, E., Local blockade of epithelial PDL-1 in the airways enhances $\mathrm{T}$ cell function and viral clearance during influenza virus infection. Journal of virology 2013, 87 (23), 12916-12924.

10. Liang, S. C.; Latchman, Y. E.; Buhlmann, J. E.; Tomczak, M. F.; Horwitz, B. H.; 
443

444

445

446

447

448

449

450

451

452

453

454

455

456

457

458

459

460

461

462

463

464

Freeman, G. J.; Sharpe, A. H., Regulation of PD-1, PD-L1, and PD-L2 expression during normal and autoimmune responses. European journal of immunology 2003, 33 (10), 2706-2716.

11. Hrdlickova, R.; Toloue, M.; Tian, B., RNA-Seq methods for transcriptome analysis. Wiley Interdisciplinary Reviews: RNA 2017, 8 (1), e1364.

12. Nagalakshmi, U.; Waern, K.; Snyder, M., RNA-Seq: a method for comprehensive transcriptome analysis. Current protocols in molecular biology 2010, 89 (1), 4.11. 1-4.11. 13.

13. Bowers, N. L.; Helton, E. S.; Huijbregts, R. P.; Goepfert, P. A.; Heath, S. L.; Hel, Z., Immune suppression by neutrophils in HIV-1 infection: role of PD-L1/PD-1 pathway. PLoS pathogens 2014, 10 (3), e1003993.

14. Day, C. L.; Kaufmann, D. E.; Kiepiela, P.; Brown, J. A.; Moodley, E. S.; Reddy, S.; Mackey, E. W.; Miller, J. D.; Leslie, A. J.; DePierres, C., PD-1 expression on HIV-specific $\mathrm{T}$ cells is associated with T-cell exhaustion and disease progression. Nature 2006, 443 (7109), 350-354.

15. Nakamoto, N.; Kaplan, D. E.; Coleclough, J.; Li, Y.; Valiga, M. E.; Kaminski, M.; Shaked, A.; Olthoff, K.; Gostick, E.; Price, D. A., Functional restoration of HCV-specific $\mathrm{CD}^{+} \mathrm{T}$ cells by PD-1 blockade is defined by PD-1 expression and compartmentalization. Gastroenterology 2008, 134 (7), 1927-1937. e2.

16. Peng, G.; Li, S.; Wu, W.; Tan, X.; Chen, Y.; Chen, Z., PD-1 upregulation is associated with HBV-specific $\mathrm{T}$ cell dysfunction in chronic hepatitis $\mathrm{B}$ patients. Molecular immunology 2008, 45 (4), 963-970. 
465

466

467

468

469

470

471

472

473

474

475

476

477

478

479

480

481

482

483

484

485

486

17. Rutigliano, J. A.; Sharma, S.; Morris, M. Y.; Oguin III, T. H.; McClaren, J. L.;

Doherty, P. C.; Thomas, P. G., Highly pathological influenza A virus infection is associated with augmented expression of PD-1 by functionally compromised virus-specific $\mathrm{CD}^{+} \mathrm{T}$ cells. Journal of virology 2014, 88 (3), 1636-1651.

18. Deurenberg, R. H.; Bathoorn, E.; Chlebowicz, M. A.; Couto, N.; Ferdous, M.; Garc"a.a-Cobos, S.; Kooistra-Smid, A. M.; Raangs, E. C.; Rosema, S.; Veloo, A. C., Application of next generation sequencing in clinical microbiology and infection prevention. Journal of biotechnology 2017, 243, 16-24.

19. Mutz, K.-O.; Heilkenbrinker, A.; L?nne, M.; Walter, J.-G.; Stahl, F., Transcriptome analysis using next-generation sequencing. Current opinion in biotechnology 2013, 24 (1), 22-30.

20. Mamas, M. A.; Fraser, D.; Neyses, L., Cardiovascular manifestations associated with influenza virus infection. International journal of cardiology 2008, 130 (3), 304-309.

21. Ukimura, A.; Satomi, H.; Ooi, Y.; Kanzaki, Y., Myocarditis associated with influenza A H1N1pdm2009. Influenza research and treatment 2012, 2012.

22. Collins, S. D., Excess mortality from causes other than influenza and pneumonia during influenza epidemics. Public Health Reports (1896-1970) 1932, 2159-2179.

23. Kodama, M., Influenza myocarditis. Circulation Journal 2010, 74 (10), 2060-2061.

24. Gupta, S.; Markham, D. W.; Drazner, M. H.; Mammen, P. P., Fulminant myocarditis. Nature clinical practice cardiovascular medicine 2008, 5 (11), 693-706. 
487

488

489

490

491

492

493

494

495

496

497

498

499

500

501

502

503

504

505

506

507

508

25. Ammirati, E.; Veronese, G.; Cipriani, M.; Moroni, F.; Garascia, A.; Brambatti, M.;

Adler, E. D.; Frigerio, M., Acute and fulminant myocarditis: a pragmatic clinical approach to diagnosis and treatment. Current cardiology reports 2018, 20 (11), 1-12.

26. Muthumani, K.; Choo, A. Y.; Shedlock, D. J.; Laddy, D. J.; Sundaram, S. G.;

Hirao, L.; Wu, L.; Thieu, K. P.; Chung, C. W.; Lankaram, K. M., HIV-1 Nef induces

Programmed Death (PD)-1 expression through a p38 MAPK dependent mechanism. Journal of virology 2008.

27. Muthumani, K.; Choo, A. Y.; Shedlock, D. J.; Laddy, D. J.; Sundaram, S. G.;

Hirao, L.; Wu, L.; Thieu, K. P.; Chung, C. W.; Lankaraman, K. M., Human immunodeficiency virus type 1 Nef induces programmed death 1 expression through a p38 mitogen-activated protein kinase-dependent mechanism. Journal of virology 2008, 82 (23), 11536-11544.

28. Lu, L. Y.; Askonas, B. A., Cross-reactivity for different type A influenza viruses of a cloned T-killer cell line. Nature 1980, 288 (5787), 164-165.

29. Lucchese, G.; Capone, G.; Kanduc, D., Peptide sharing between influenza A H1N1 hemagglutinin and human axon guidance proteins. Schizophrenia bulletin 2014, $40(2), 362-375$.

30. Wiwanitkit, S.; Wiwanitkit, V., Influenza A H1N1 hemagglutinin and human axon guidance proteins: Peptide sharing but not same epitopes. Indian journal of human genetics 2013, 19 (4), 512.

31. Azoulay-Alfaguter, I.; Strazza, M.; Pedoeem, A.; Mor, A., The coreceptor programmed death 1 inhibits T-cell adhesion by regulating Rap1. Journal of Allergy 
510 32. Quiles, J. M.; Narasimhan, M.; Shanmugam, G.; Mosbruger, T.; Rajasekaran, N. 511 S., A novel microRNA signature in myocardial reductive stress. Free Radical Biology 512 and Medicine 2016, 100, S149.

513 33. Olingy, C., Rewiring aged T cells. American Association for the Advancement of 514 Science: 2021. 